\title{
Distinct IncRNA transcriptional fingerprints characterize progressive stages of multiple myeloma
}

\author{
Domenica Ronchetti ${ }^{1,2, *}$, Luca Agnelli ${ }^{1,2, *}$, Elisa Taiana ${ }^{1,2}$, Serena Galletti ${ }^{1,2}$, Martina \\ Manzoni ${ }^{1,2}$, Katia Todoerti ${ }^{3}$, Pellegrino Musto ${ }^{3}$, Francesco Strozzi ${ }^{4}$, Antonino Neri ${ }^{1,2}$ \\ ${ }^{1}$ Department of Oncology and Hemato-Oncology, University of Milano, Milan, Italy \\ ${ }^{2}$ Hematology Unit, Fondazione IRCCS Ca' Granda, Ospedale Maggiore Policlinico, Milan, Italy \\ ${ }^{3}$ Laboratory of Pre-Clinical and Translational Research, IRCCS-CROB, Referral Cancer Center of Basilicata, Rionero in \\ Vulture, Potenza, Italy \\ ${ }^{4}$ Bioinformatics Core Facility, Parco Tecnologico Padano, Lodi, Italy \\ ${ }^{*}$ These authors have contributed equally to this work \\ Correspondence to: Antonino Neri, e-mail: antonino.neri@unimi.it \\ Luca Agnelli, e-mail: luca.agnelli@gmail.com \\ Keywords: IncRNA, multiple myeloma, plasma cell dyscrasia, MALATI, expression profiling \\ Received: November 02, $2015 \quad$ Accepted: January 29, $2016 \quad$ Published: February 17, 2016
}

\section{ABSTRACT}

Although many efforts have recently contributed to improve our knowledge of molecular pathogenesis of multiple myeloma (MM), the role and significance of long non-coding RNAs (IncRNAs) in plasma cells (PC) malignancies remains virtually absent. To this aim, we developed a custom annotation pipeline of microarray data investigating IncRNA expression in PCs from 20 monoclonal gammopathies of undetermined significance, 33 smoldering MM, 170 MM, and 36 extra-medullary MMs/plasma cell leukemia patients, and 9 healthy donors. Our study identified 31 IncRNAs deregulated in tumor samples compared to normal controls; among these, the upregulation of MALAT1 appeared associated in MM patients with molecular pathways involving cell cycle regulation, p53-mediated DNA damage response, and mRNA maturation processes. Furthermore, we found 21 IncRNAs whose expression were progressively deregulated trough the more aggressive stages of PC dyscrasia, suggesting a possible role in the progression of the disease. Finally, in the context of molecular heterogeneity of MM, we identified a transcriptional fingerprint in hyperdiploid patients, characterized by the upregulation of IncRNAs/pseudogenes related to ribosomal protein genes, known to be upregulated in this molecular group. Overall, the data provides an important resource for future studies on the functions of IncRNAs in the pathology.

\section{INTRODUCTION}

Multiple myeloma (MM) is a malignant proliferation of antibody-secreting bone marrow plasma cells (PCs) characterized by a wide clinical spectrum ranging from the presumed pre-malignant condition called monoclonal gammopathy of undetermined significance (MGUS), to smoldering MM (SMM), truly overt and symptomatic MM, and extra-medullary myeloma/plasma cell leukemia (PCL) [1-3]. Despite the remarkable improvements in treatment and patient care [4], MM remains an incurable disease.

During the years that have followed human genome sequencing, it has become evident that over $90 \%$ of the genome is actively transcribed $[5,6]$, the majority of transcripts being represented by non-coding RNA (ncRNA) and therefore not translated into proteins. NcRNAs are broadly divided into short $(<200 \mathrm{nt})$ and long (>200 nt) transcripts. Dysregulation of short ncRNAs, particularly miRNAs, has been reported to occur virtually in all types of cancer, including MM, highlighting the usefulness of miRNA profiling in diagnosis, prognosis, and in predicting response to therapy [7, 8]. Notably, miRNAs are currently considered both emerging therapeutic targets and innovative intervention tools in cancer including MM [9-11].

Long non-coding RNAs (lncRNAs) are a very heterogeneous group that lack mRNA properties, although they exhibit a structure and biogenesis that does not differ 
greatly from mRNAs: indeed, they can be polyadenylated and may operate in either nuclear and/or cytoplasmic fractions. LncRNAs represent more than half of the mammalian noncoding transcriptome and are involved in many biological processes, such as transcriptional gene regulation, maintenance of genomic integrity, $\mathrm{X}$-chromosome inactivation, genomic imprinting, cell differentiation, and development [12]. Among lncRNAs, there are also pseudogenes that have lost their proteincoding ability or are otherwise no longer translated; however, they may be functional, similar to other kinds of non-coding RNA, and can have a regulatory role [13]. LncRNAs can also be classified based on their location relative to nearby protein-coding genes. They originate from introns, exons, intergenic, promoter regions, 3'and 5'-UTRs; therefore they may represent antisense, bidirectional, or sense-overlapping sequences of specific genes $[14,15]$. Several lncRNAs exhibit temporal and spatial expression patterns; moreover, their expression can be restricted to particular tissue or cell cycle stages, indicating diverse biological roles for lncRNAs [16]. Deregulation of distinct lncRNAs has been reported to promote tumor formation, progression, and metastasis in many types of cancer, including hematologic malignancies $[17,18]$. The number of known human IncRNA transcripts is still evolving. LNCipedia v3.1 is the largest integrated repository containing 111,685 human annotated lncRNAs, with many loci generating multiple transcripts [19].

The knowledge of the role of lncRNAs in MM is virtually absent. A recent paper investigated lncRNAs as biomarkers for predicting survival in MM patients [20]; in addition, MALAT1 (metastasis-associated lung adenocarcinoma transcript 1), a putative oncogenic IncRNA overexpressed in several solid tumors [21, 22], has been found overexpressed in $\mathrm{MM}$ and may represent a marker to predict MM progression [23]. Moreover, it is expressed in bone marrow mononuclear cells of newly diagnosed myeloma patients [23]. Finally, preliminary evidence has suggested deregulated levels of circulating lncRNAs in MM patients compared to healthy donors [24].

The present study was aimed at investigating the lncRNA expression profiles in a large and representative cohort of PC dyscrasia, including MGUS, SMM, MM, and PCL patients, and in normal bone marrow PC controls. We have identified deregulated lncRNAs putatively associated with MM pathogenesis and possibly linked to the accepted multi-step process underlining the different stages of the disease.

\section{RESULTS}

\section{LncRNA expression profiling in plasma cell dyscrasia}

The expression profiles of lncRNAs have been investigated by Gene 1.0 ST array in a large cohort of 268 patients included in two different datasets and representative of the major forms of plasma cell dyscrasia. The panel totally included 20 MGUS, 33 SMM, 170 MM, 36 PCL patients and 9 normal bone marrow PCs samples. To be confident in detecting specifically lncRNAs, we applied a custom annotation pipeline able to remap the probes included in the original array to distinct lncRNAs according to the LNCipedia-v3.1 database (see Methods and Figure 1). Such a strategy allowed us to investigate the expression levels of 1852 well-annotated and specific human IncRNAs.

To determine whether the natural grouping of lncRNAs expression profiling could be associated with a specific clinical entity, we performed an unsupervised analysis using conventional hierarchical agglomerative clustering of the 230 lncRNAs whose average change in expression levels varied at least 1.5 -fold from the mean across the dataset (Figure 2). Notably, we found that all normal controls clustered together (cluster in the green box, $P<0.0001$ ), as did 19 of 20 MGUS cases (cluster in the blue box, $P=0.0011)$ and SMM patients $(P=0.0049)$. Notably, the lncRNAs strongly upregulated in normal samples (Figure 2, black box) are located in chromosomal regions, such as $14 q 32,2 p$, and $22 p$, coding for the highly variable portions of the immunoglobulin genes or for IGV pseudogenes. We related this to the highly complex transcriptional activity of these regions in normal PCs to achieve polyclonal antibody repertoire, therefore excluded these transcripts from further analyses.

Next, we compared normal samples with MGUS, SMM, MM or PCL patients, and identified a set of 160 lncRNAs showing highly significant differential expression between normal PCs and those from the four different clinical entities (Supplementary Table S1). In particular, among this lncRNA panel, six transcripts were common to all the comparisons, whereas 25 lncRNAs resulted from at least three analyses (Table 1). Among these $31 \operatorname{lncRNAs,} 19$ were upregulated in the pathological samples compared to normal plasma cells; notably lnc-SCYL1-1, also known as MALAT1. This group also includes lnc-MON2-2, lnc-PTPDC1-7, and lnc-USP25-2, all of which overlapping host genes for members of the let-7 miRNA family, and lnc-USP25-2, lncAPC6, lnc-LIPG-3, lnc-TRPV2-1 and lnc-C3orf25-2 that overlap host genes for small nucleolar RNAs (snoRNAs) molecules. Among the 19 upregulated lncRNAs, we found seven transcripts classified in Ensembl as pseudogenes; for each $\operatorname{lncRNA} /$ pseudogene, we looked for the corresponding putative parental gene when identifiable by the blat search (UCSC Blat tool at http://genome.ucsc. edu/index.html). Specifically, as shown in Table 1, lncC3orf25-2, lnc-MC2R-2, lnc-CHRDL2-3, lnc-SYT8-3, lnc-PAIP1-3, and lnc-ABCB5-3, are all pseudogenes of ribosomal protein encoding genes, whereas lnc-SAFB2-3 is a pseudogene of the SNRPE gene, which encodes a protein belonging to the large family of small nuclear 
ribonucleoproteins ( $\mathrm{snRNPs}$ ), the building blocks of the spliceosome complex. In addition, we found a significant upregulation of lnc-ANGPTL1-3 and lnc-RLIM-6 that map antisense to the Ras-specific guanine nucleotidereleasing factor RALGPS2 and to the thyroid hormone transporter SLC16A2, respectively; and lnc-ARFIP1-5 localizing antisense to the E3 ubiquitin ligase $F B X W 7$ gene but also overlapping the MIR4453 locus. Finally, lncSENP5-4 and lnc-AP1M2-1 localize head to head to the nuclear cap binding protein $N C B P 2$ gene, and interleukin enhancer binding factor 3 (ILF3), respectively. Among the 12 lncRNAs downregulated in the pathological samples compared to normal plasma cells, we found lnc-SNURF-1 and lnc-SNURF-3 that are host genes for SNORD115 and
SNORD116 families. Two pseudogenes of the RN7SL gene at $14 \mathrm{q} 21$ were found also downregulated. This gene encodes the small cytoplasmic RNA component of the signal recognition particle (SRP) that associates with the ribosome and targets nascent proteins to the endoplasmic reticulum for secretion or membrane insertion. Other four pseudogenes are included in the downregulated group: Inc-STOM-7 that is a remnant of the Alpha-1,3galactosyltransferase (GGTA1) gene encoding an enzyme present in most mammals except man; lnc-HSFY2-10 that is positioned in sense orientation to its parental CD24 gene; lnc-CISH-3, and lnc-SEL1L3-6. Furthermore, we found the down-regulation of lnc-LRRC47-1, described below.

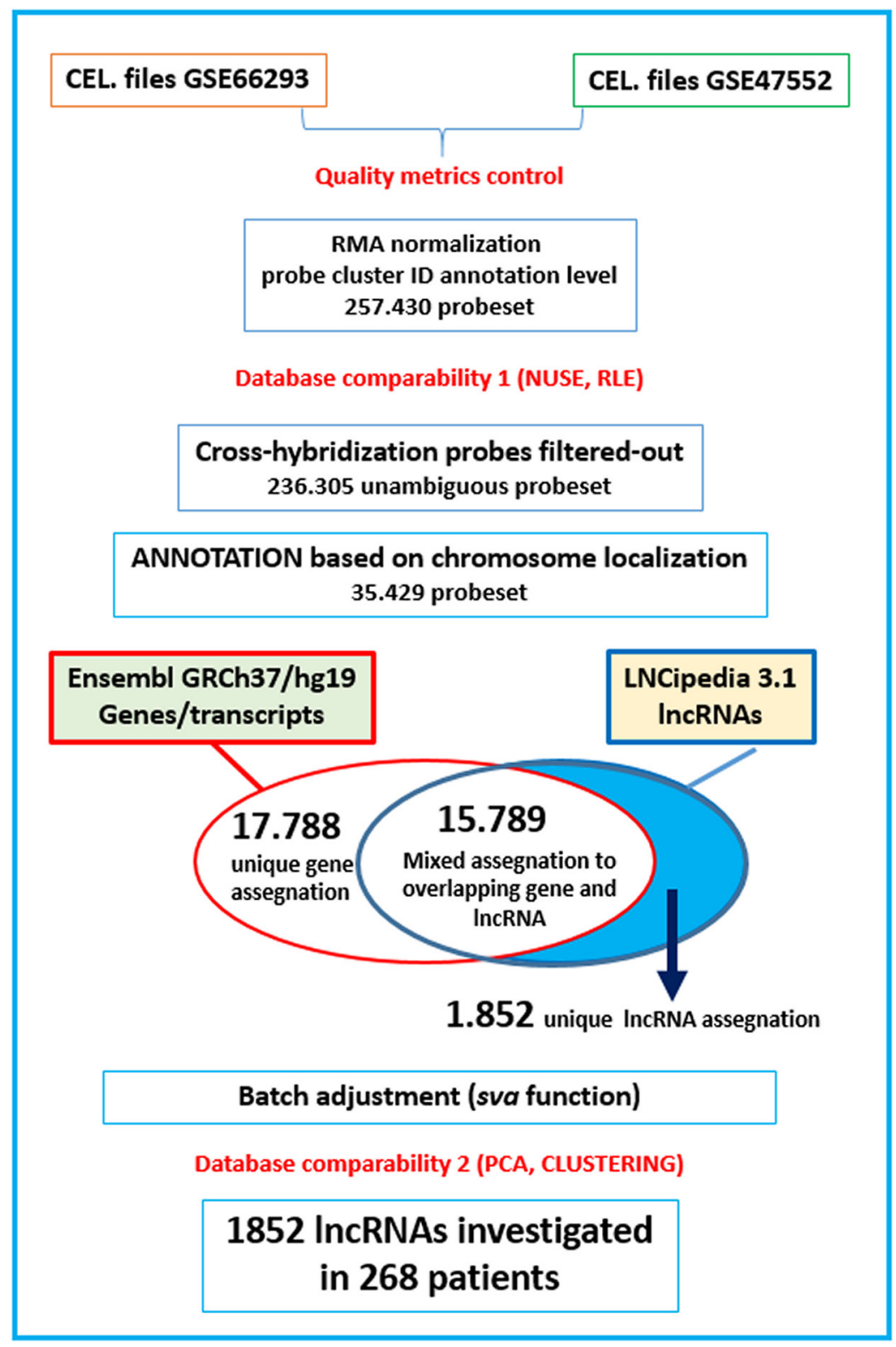

Figure 1: Custom annotation pipeline. Flow diagram highlighting the different steps for processing the Gene $1.0 \mathrm{ST}$ array data to investigate lncRNAs in combined databases (GSE66293 and GSE47552). 


\section{LncRNA expression in the different stages of the disease}

In order to identify deregulated lncRNAs possibly associated with the different clinico-biological forms of the disease, we searched for lncRNAs whose expression was significantly and progressively increased/decreased from normal PCs to PCLs (Jonckheere-Terpstra test). As reported in Table 2 and Supplementary Figure S1, the expression levels of 15 lncRNAs progressively increased from normal to MGUS, SMM, MM and PCL samples. On the other hand, six lncRNAs displayed a significantly decreasing trend. From a structural point of view, the resulting 21 lncRNAs could be divided in two main categories: i.e. (i) lncRNAs that are transcribed as complex, interlaced networks of overlapping transcripts often including protein-coding genes (as specified in Table 2 , fourth column); and (ii) those located and transcribed within the intergenic stretches of the genome.

Representative of the first group was lnc-LRRC47-1, whose expression showed a significant decreasing trend being significantly downregulated in MGUS, MM and PCL samples as compared to normal control (see above). In details, lnc-LRRC47-1 could be transcribed in 23 different transcripts, seven of which correspond to the reported TP73-AS1 transcripts that map antisense to the 3'UTR of the tumor suppressor TP73 gene. In agreement with the previous analyses, we found a significant increasing expression trend of lnc-ANGPTL1-3, lncRLIM-6, and lnc-SENP5-4 from normal to pathological samples. Among the first group, we have also identified lnc-WHAMM-2 and lnc-SERPINC1-1, also known as SNHG21 and GAS5, respectively, which are host genes for SNORD molecules, and both showing a significant positive expression trend. Finally, this group also included three pseudogenes, the above described lnc-HSFY2-10, lnc-SNX29P2-3 that maps antisense to the NPIPL1 gene encoding a nuclear pore complex-interacting protein member, and lnc-CPSF2-2, which is a pseudogene of Prothymosin alpha (PTMA) and is localized antisense to the thyroid hormone receptor interactor 11 (TRIP11) gene.

The second lncRNA group included 12 transcripts 10 of which are pseudogenes. Among the nine with a significant increasing expression trend from normal PCs to PCLs, we found lnc-PNRC1-1, lnc-DLX5-4, and lncZC3H12B-10, which are all pseudogenes of the RN7SL gene. Lnc-SAFB2-3 and lnc-KIF20B-7 are pseudogenes of $S N R P E$ and $S N R P D 2$, respectively, which encode proteins included in the snRNPs; notably, the expression levels of these lncRNAs resulted significantly correlated with those of corresponding parental genes (Table 2, last column). Lnc-MC2R-2 and lnc-DNAJC16-1 are respectively processed pseudogenes of RPL36A gene and the transcription factor CHCHD2 that transactivates a conserved oxygen response element. Moreover, lncWDR11-7 is a pseudogene of the RN7SK family genes, involved in RNA Polymerase II activity control.

Finally, we investigated whether the lncRNAs that were gradually deregulated through the progressive stages of PC dyscrasia were also related to the disease progression in the same patient. To this regard, Gene Expression Profiling (GEP) data from a limited proprietary paired cohort of 19 MM patients at diagnosis and relapse/PCL progression corroborated the deregulated expression of lnc-SENP5-4, lnc-CPSF2-2, and lnc-LRRC47-1 during disease progression in this subset of patients (Supplementary Figure S2).

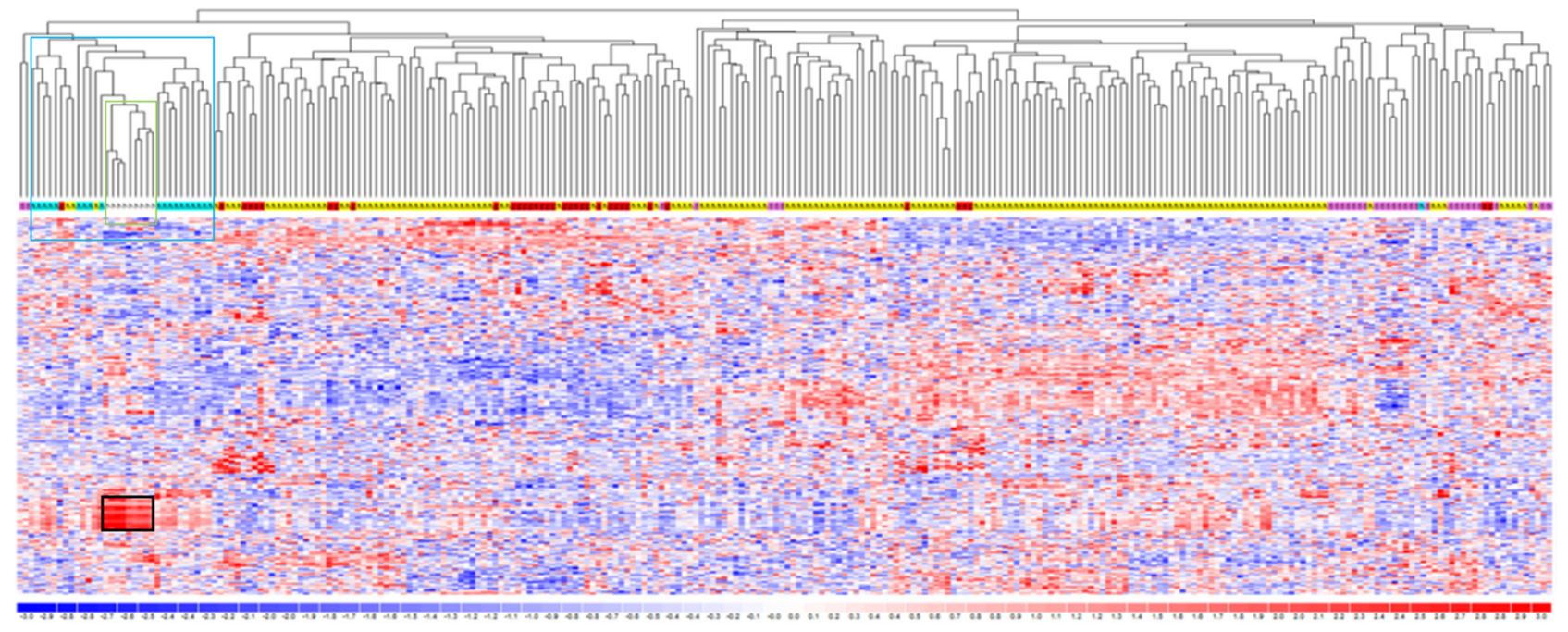

Figure 2: LncRNA expression profiling in plasma cell dyscrasia. Hierarchical clustering of the 268 samples using the 230 most variable lncRNAs (patients in columns, lncRNAs in rows). The color scale bar represents the relative lncRNA expression changes normalized by the standard deviation. Color above the matrix indicates the type of samples: white, light blue, pink, yellow, and red represent Normal (N), MGUS, SMM, MM, and PCL samples, respectively. Specific types are enriched in colored sub-branches (see text). Black box identifies lncRNAs strongly upregulated in normal samples (see text). 
Table 1: Differentially expressed LncRNAs resulting from SAM analyses comparing N with MGUS, sMM, MM, or PCL patients

\begin{tabular}{|c|c|c|c|c|c|c|c|c|c|c|c|}
\hline $\operatorname{lncRNA}$ & z & 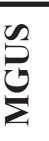 & $\sum_{\infty}$ & $\sum$ & $\vec{U}$ & Chr & $\begin{array}{c}\text { ALIAS; } \\
\text { OVERLAPPING } \\
\text { TRANSCRIPT }^{a}\end{array}$ & $\begin{array}{c}\text { Corr. } \\
\text { IncRNA- } \\
\text { transcript }^{b}\end{array}$ & PsG $^{\mathrm{c}}$ & $\begin{array}{c}\text { Putative } \\
\text { Parental } \\
\text { Gene }\end{array}$ & $\begin{array}{l}\text { Corr. IncRNA- } \\
\text { parental gene }\end{array}$ \\
\hline AC100793.1-1 & $\uparrow$ & $\downarrow$ & $\downarrow$ & $\downarrow$ & $\downarrow$ & $17 \mathrm{q} 21$ & RAMP2-AS1 & & & & \\
\hline ATL3-1 & $\uparrow$ & $\downarrow$ & $\downarrow$ & $\downarrow$ & $\downarrow$ & $11 \mathrm{q} 13$ & C11orf95 & & & & \\
\hline ADAP2-2 & $\uparrow$ & $\downarrow$ & $\downarrow$ & $\downarrow$ & $\downarrow$ & $17 \mathrm{q} 11$ & RN7SL138P & & M & $\begin{array}{l}\text { RN7SL } \\
(14 \mathrm{q} 21)\end{array}$ & NA \\
\hline STOM-7 & $\uparrow$ & & $\downarrow$ & $\downarrow$ & $\downarrow$ & $9 \mathrm{q} 33$ & GGTA1P & & UP & & \\
\hline HSFY2-10 & $\uparrow$ & & $\downarrow$ & $\downarrow$ & $\downarrow$ & Yq11 & $\begin{array}{l}\text { CD24P4; } \mathrm{S} \text { to } \\
\mathrm{CD} 24\end{array}$ & NA & PP & $\begin{array}{l}\text { CD24 } \\
\text { (Yq11) }\end{array}$ & NA \\
\hline CISH-3 & $\uparrow$ & $\downarrow$ & & $\downarrow$ & $\downarrow$ & $3 \mathrm{p} 21$ & $\begin{array}{c}\text { ZNF652P1; AS to } \\
\text { DOCK3 }\end{array}$ & $\mathrm{R}=0.053$ & PP & $\begin{array}{l}\text { ZNF652 } \\
(17 q 21)\end{array}$ & $\mathrm{R}=0.65 \mathrm{p}<2.2 \mathrm{e}-16$ \\
\hline LRRC47-1 & $\uparrow$ & $\downarrow$ & & $\downarrow$ & $\downarrow$ & $1 \mathrm{p} 36$ & $\begin{array}{c}\text { TP73-AS1; AS to } \\
\text { TP73 }\end{array}$ & $\mathrm{R}=0.03$ & & & \\
\hline SEL1L3-6 & $\uparrow$ & $\downarrow$ & $\downarrow$ & & $\downarrow$ & $4 \mathrm{p} 15$ & & & $\mathrm{UnP}$ & NA & \\
\hline SEMA4B-4 & $\uparrow$ & $\downarrow$ & $\downarrow$ & & $\downarrow$ & $15 \mathrm{q} 26$ & & & & & \\
\hline PQLC2-5 & $\uparrow$ & $\downarrow$ & $\downarrow$ & & $\downarrow$ & $1 \mathrm{p} 36$ & $\begin{array}{l}\text { RN7SL277P; AS } \\
\text { to CAPZB }\end{array}$ & $R=-0.26$ & M & $\begin{array}{l}\text { RN7SL } \\
(14 \mathrm{q} 21)\end{array}$ & NA \\
\hline SNURF-1 & $\uparrow$ & $\downarrow$ & $\downarrow$ & & $\downarrow$ & $15 q 11$ & $\begin{array}{l}\text { S to } \\
\text { SNORD115-116 } \\
\text { family }\end{array}$ & NA & & & \\
\hline SNURF-3 & $\uparrow$ & $\downarrow$ & $\downarrow$ & & $\downarrow$ & $15 \mathrm{q} 11$ & $\begin{array}{l}\text { S to } \\
\text { SNORD115-116 } \\
\text { family }\end{array}$ & NA & & & \\
\hline APC-6 & $\downarrow$ & $\uparrow$ & $\uparrow$ & $\uparrow$ & $\uparrow$ & $5 \mathrm{q} 22$ & $\begin{array}{l}\text { EPB41L4A-AS1; } \\
\text { S to SNORA13 }\end{array}$ & NA & & & \\
\hline MON2-2 & $\downarrow$ & $\uparrow$ & $\uparrow$ & $\uparrow$ & $\uparrow$ & $12 \mathrm{q} 14$ & S to Let7i & NA & & & \\
\hline ARFIP1-5 & $\downarrow$ & $\uparrow$ & $\uparrow$ & $\uparrow$ & $\uparrow$ & $4 q 13$ & $\begin{array}{l}\text { AS to FBXW7, S } \\
\text { to MIR4453 }\end{array}$ & $\begin{array}{c}\mathrm{R}=0.03 \\
\text { (FBXW7) }\end{array}$ & & & \\
\hline PTPDC1-7 & $\downarrow$ & & $\uparrow$ & $\uparrow$ & $\uparrow$ & $9 q 22$ & $\begin{array}{c}\text { AS to } \\
\text { MIRLET7DHG }\end{array}$ & NA & & & \\
\hline MC2R-2 & $\downarrow$ & & $\uparrow$ & $\uparrow$ & $\uparrow$ & $18 \mathrm{p} 11$ & & & PP & $\begin{array}{c}\text { RPL36A } \\
\text { (Xq22) }\end{array}$ & NA \\
\hline SAFB2-3 & $\downarrow$ & & $\uparrow$ & $\uparrow$ & $\uparrow$ & $19 \mathrm{p} 13$ & SNRPEP4 & & PP & $\begin{array}{l}\text { SNRPE } \\
(1 \mathrm{q} 32)\end{array}$ & $\begin{array}{c}\mathrm{R}=0.5 \\
\mathrm{p}=0.030\end{array}$ \\
\hline SYT8-3 & $\downarrow$ & & $\uparrow$ & $\uparrow$ & $\uparrow$ & $11 \mathrm{p} 15$ & RPL36AP39 & & PP & $\begin{array}{c}\text { RPL36A } \\
\text { (Xq22) }\end{array}$ & NA \\
\hline CHRDL2-3 & $\downarrow$ & & $\uparrow$ & $\uparrow$ & $\uparrow$ & $11 \mathrm{q} 13$ & & & PP & $\begin{array}{l}\text { RPS12 } \\
\text { (6q23) }\end{array}$ & $\mathrm{R}=0.17$ \\
\hline SCYL1-1 & $\downarrow$ & & $\uparrow$ & $\uparrow$ & $\uparrow$ & $11 \mathrm{q} 13$ & MALAT1 & & & & \\
\hline
\end{tabular}




\begin{tabular}{|c|c|c|c|c|c|c|c|c|c|c|}
\hline IncRNA & 乙 & $\sum_{\infty}$ & $\sum$ & $\vec{U}$ & Chr & $\begin{array}{c}\text { ALIAS; } \\
\text { OVERLAPPING } \\
\text { TRANSCRIPT }^{a}\end{array}$ & $\begin{array}{c}\text { Corr. } \\
\text { IncRNA- } \\
\text { transcript }\end{array}$ & $\mathbf{P s G}^{\mathrm{c}}$ & $\begin{array}{c}\text { Putative } \\
\text { Parental } \\
\text { Gene }\end{array}$ & $\begin{array}{l}\text { Corr. IncRNA- } \\
\text { parental gene }\end{array}$ \\
\hline TRPV2-1 & $\downarrow$ & $\uparrow$ & $\uparrow$ & $\uparrow$ & $17 \mathrm{p} 11$ & $\begin{array}{l}\text { LRRC75A-AS1; } \\
\text { S to SNORD49B, } \\
\quad 49 \mathrm{~A}, 65\end{array}$ & NA & & & \\
\hline LIPG-3 & $\downarrow$ & $\uparrow$ & $\uparrow$ & $\uparrow$ & $18 \mathrm{q} 21$ & SNHG22 & & & & \\
\hline USP25-2 & $\downarrow$ & $\uparrow$ & $\uparrow$ & $\uparrow$ & $21 \mathrm{q} 21$ & $\begin{array}{l}\text { S to SNORD74, } \\
\text { MIR99AHG }\end{array}$ & NA & & & \\
\hline C3orf25-2 & $\downarrow$ & $\uparrow$ & $\uparrow$ & $\uparrow$ & $3 q 21$ & $\begin{array}{l}\text { RPL32P3; S to } \\
\text { SNORA7B }\end{array}$ & NA & UnP & $\begin{array}{l}\text { RPL32 } \\
\text { (3p25) }\end{array}$ & $\mathrm{R}=0.47 \mathrm{p}<2.2 \mathrm{e}-16$ \\
\hline AP1M2-1 & $\downarrow$ & $\uparrow$ & $\uparrow$ & $\uparrow$ & $19 \mathrm{p} 13$ & ILF3-AS1 & & & & \\
\hline ANGPTL1-3 & $\downarrow$ & $\uparrow$ & $\uparrow$ & $\uparrow$ & $1 \mathrm{q} 25$ & AS to RALGPS2 & $\begin{array}{c}\mathrm{R}=0.3 \\
\mathrm{p}=3.8 \mathrm{e}-08\end{array}$ & & & \\
\hline SENP5-4 & $\downarrow$ & $\uparrow$ & $\uparrow$ & $\uparrow$ & $3 q 29$ & NCBP2 -AS2 & & & & \\
\hline PAIP1-3 & $\downarrow$ & $\uparrow$ & $\uparrow$ & $\uparrow$ & $5 \mathrm{p} 12$ & $\begin{array}{c}\text { RPL29P12; AS to } \\
\text { NNT }\end{array}$ & $\mathrm{R}=-0.054$ & PP & $\begin{array}{l}\text { RPL29 } \\
\text { (3p21) }\end{array}$ & $\mathrm{R}=0.7 \mathrm{p}<2.2 \mathrm{e}-16$ \\
\hline ABCB5-3 & $\downarrow$ & $\uparrow$ & $\uparrow$ & $\uparrow$ & $7 \mathrm{p} 21$ & RPL23P8 & & PP & $\begin{array}{l}\text { RPL23 } \\
(17 \mathrm{q} 12)\end{array}$ & NA \\
\hline RLIM-6 & $\downarrow$ & $\uparrow$ & $\uparrow$ & $\uparrow$ & $\mathrm{Xq13}$ & AS to SLC16A2 & $\begin{array}{c}\mathrm{R}=0.37 \\
\mathrm{p}=3.7 \mathrm{e}-10\end{array}$ & & & \\
\hline
\end{tabular}

Arrows indicate lncRNA expression, upregulation $(\uparrow)$ or downregulation $(\downarrow)$, in the corresponding group. Chromosomal localization $(\mathrm{Chr})$, alias name, and overlapping transcripts are indicated.

${ }^{\text {a }}$ Sense (S) to, or Antisense (AS) to overlapping transcripts; ${ }^{\mathrm{b}}$ Pearson correlation coefficient; NA $=$ not available (not detected by the array); ${ }^{\mathrm{P} s \mathrm{G}}=\mathrm{Pseudogene}$; Ensembl type: $\mathrm{PP}=$ Processed Pseudogene; UP= Unitary Pseudogene; $\mathrm{UnP}=$ Unprocessed Pseudogene; $\mathrm{M}=$ miscellaneous RNA.

Table 2: LncRNAs significantly increased/decreased in the progressive forms of plasma cell dyscrasias

\section{InCRNAs SENSE or ANTISENSE (S or AS) TO ANNOTATED TRANSCRIPTS}

\begin{tabular}{|c|c|c|c|c|c|c|c|}
\hline 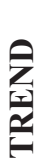 & $\begin{array}{c}\text { IncRNAs } \\
\mathbf{q}<0.05^{\mathrm{a}}\end{array}$ & Chr & $\begin{array}{c}\text { ALIAS; } \\
\text { OVERLAPPING } \\
\text { TRANSCRIPTS }^{\mathrm{b}}\end{array}$ & $\begin{array}{c}\text { Corr. } \\
\text { IncRNA- } \\
\text { overlapping } \\
\text { transcript }^{\mathrm{c}}\end{array}$ & $\mathbf{P s G}^{\mathrm{d}}$ & $\begin{array}{c}\text { Putative } \\
\text { Parental } \\
\text { Gene }\end{array}$ & $\begin{array}{l}\text { Corr. IncRNA- } \\
\text { parental gene }\end{array}$ \\
\hline$\uparrow$ & RLIM-6 & $\mathrm{Xq13}$ & AS to SLC16A2 & $\begin{array}{c}\mathrm{R}=0.37 \\
\mathrm{p}=3.7 \mathrm{e}-10\end{array}$ & & & \\
\hline$\uparrow$ & ANGPTL1-3 & $1 \mathrm{q} 25$ & AS to RALGPS2 & $\begin{array}{c}\mathrm{R}=0.3 \\
\mathrm{p}=3.8 \mathrm{e}-08\end{array}$ & & & \\
\hline$\uparrow$ & SENP5-4 & $3 q 29$ & $\begin{array}{l}\text { NCBP2-AS2; } \mathrm{S} \text { to } \\
\text { NCBP2 }\end{array}$ & $\mathrm{R}=-0.054$ & & & \\
\hline$\downarrow$ & LRRC47-1 & $1 \mathrm{p} 36$ & $\begin{array}{c}\text { TP73-AS1; AS to } \\
\text { TP73 }\end{array}$ & $\mathrm{R}=0.03$ & & & \\
\hline$\uparrow$ & WHAMM-2 & $15 q 25$ & $\begin{array}{c}\text { SNHG21; AS to } \\
\text { FSD2 }\end{array}$ & $R=-0.03$ & & & \\
\hline
\end{tabular}

(continued) 
IncRNAs SENSE or ANTISENSE (S or AS) TO ANNOTATED TRANSCRIPTS

\begin{tabular}{|c|c|c|c|c|c|c|c|}
\hline 网 & $\begin{array}{c}\text { IncRNAs } \\
\mathbf{q}<0.05^{\mathrm{a}}\end{array}$ & Chr & $\begin{array}{c}\text { ALIAS; } \\
\text { OVERLAPPING } \\
\text { TRANSCRIPTS }^{b}\end{array}$ & $\begin{array}{c}\text { Corr. } \\
\text { IncRNA- } \\
\text { overlapping }^{\text {transcript }} \\
\text { transing }^{\mathrm{c}}\end{array}$ & $\mathbf{P s G}^{\mathrm{d}}$ & $\begin{array}{c}\text { Putative } \\
\text { Parental } \\
\text { Gene }\end{array}$ & $\begin{array}{l}\text { Corr. IncRNA- } \\
\text { parental gene }\end{array}$ \\
\hline$\uparrow$ & 1SERPINC1-1 & $1 \mathrm{q} 25$ & $\begin{array}{l}\text { GAS5; AS to } \\
\text { ZBTB37 }\end{array}$ & $\begin{array}{c}\mathrm{R}=0.29 \\
\mathrm{p}=1.1 \mathrm{e}-06\end{array}$ & & & \\
\hline$\downarrow$ & SNX29P2-3 & $16 \mathrm{p} 11$ & AS to NPIPL1 & NA & $\mathrm{P}$ & NA & \\
\hline$\downarrow$ & HSFY2-10 & Yq11 & $\begin{array}{l}\text { CD24P4; S to } \\
\text { CD24 }\end{array}$ & NA & PP & $\begin{array}{l}\text { CD24 } \\
\text { (Yq11) }\end{array}$ & NA \\
\hline \multirow[t]{2}{*}{$\uparrow$} & CPSF2-2 & $14 q 32$ & AS to TRIP11 & $\mathrm{R}=0.063$ & PP & $\begin{array}{l}\text { PTMA } \\
(2 \mathrm{q} 37)\end{array}$ & $\begin{array}{l}R=-0.32 \\
p=7.8 e-08\end{array}$ \\
\hline & & & LINC & & & & \\
\hline 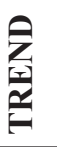 & $\begin{array}{c}\text { IncRNAs } \\
\mathbf{q}<0.05^{a}\end{array}$ & Chr & ALIAS & & $\mathbf{P s G}^{\mathrm{d}}$ & $\begin{array}{c}\text { Putative } \\
\text { Parental } \\
\text { Gene }\end{array}$ & $\begin{array}{l}\text { Corr. IncRNA- } \\
\text { parental gene }\end{array}$ \\
\hline$\downarrow$ & IRF2-3 & $4 q 35$ & & & & & \\
\hline$\downarrow$ & VKORC1L1-3 & $7 q 11$ & RP13-254B10.1 & & PP & NA & \\
\hline$\downarrow$ & STOM-7 & $9 \mathrm{q} 33$ & GGTA1P & & UP & NA & \\
\hline$\uparrow$ & RALGAPB-1 & $20 \mathrm{q} 11$ & SNHG11 & & & & \\
\hline$\uparrow$ & MC2R-2 & $18 \mathrm{p} 11$ & RP11-681N23.1 & & PP & $\begin{array}{l}\text { RPL36A } \\
\text { (Xq22) }\end{array}$ & NA \\
\hline$\uparrow$ & SAFB2-3 & $19 \mathrm{p} 13$ & SNRPEP4 & & PP & $\begin{array}{l}\text { SNRPE } \\
(1 \mathrm{q} 32)\end{array}$ & $\mathrm{R}=0.5 \mathrm{p}=0.030$ \\
\hline$\uparrow$ & KIF20B-7 & $10 \mathrm{q} 23$ & SNRPD2P1 & & PP & $\begin{array}{l}\text { SNRPD2 } \\
(19 \mathrm{q} 13)\end{array}$ & $R=0.79 p<2.2 e-16$ \\
\hline$\uparrow$ & DNAJC16-1 & $1 \mathrm{p} 36$ & $\begin{array}{c}\text { CHCHD2P6 } \\
\text { (447bp, and 462bp) }\end{array}$ & & PP & $\begin{array}{c}\text { CHCHD2 } \\
(7 \mathrm{p} 11)\end{array}$ & NA \\
\hline$\uparrow$ & WDR11-7 & $10 \mathrm{q} 26$ & RN7SKP167 RNA & & M & $\begin{array}{c}\text { RN7SK } \\
(6 \mathrm{p} 12)\end{array}$ & NA \\
\hline$\uparrow$ & PNRC1-1 & $6 q 15$ & RN7SL336P & & M & $\begin{array}{l}\text { RN7SL } \\
(14 \mathrm{q} 21)\end{array}$ & NA \\
\hline$\uparrow$ & DLX5-4 & $7 \mathrm{q} 21$ & RN7SL252P & & M & $\begin{array}{l}\text { RN7SL } \\
(14 \mathrm{q} 21)\end{array}$ & NA \\
\hline$\uparrow$ & ZC3H12B-10 & $\mathrm{Xq11}$ & RN7SL799P & & M & $\begin{array}{l}\text { RN7SL } \\
(14 \mathrm{q} 21)\end{array}$ & NA \\
\hline
\end{tabular}

Arrows indicate lncRNA expression (upregulation $\uparrow$ /downregulation $\downarrow$ ), from N to PCL samples. Chromosomal localization (Chr), alias name, and overlapping transcripts are indicated.

${ }^{a}$ IncRNAs also found in Table 1 are in bold; ${ }^{b}$ Sense (S) to, or Antisense (AS) to overlapping transcripts; ${ }^{\mathrm{c}}$ Pearson correlation coefficient; $\mathrm{NA}=$ not available (not detected by the array); ${ }^{\mathrm{d}} \mathrm{PsG}=\mathrm{Pseudogene}$; Ensembl type: $\mathrm{PP}=\mathrm{Processed}$ Pseudogene; UP= Unitary Pseudogene; UnP= Unprocessed Pseudogene; $\mathrm{M}=$ miscellaneous RNA. 


\section{LncRNA expression profiling in MM molecular subgroups}

Based on the commonly accepted notion of the great heterogeneity in MM patients, we aimed at identifying lncRNAs deregulation distinctive of the major MM molecular subgroups. We focused on the proprietary panel of $129 \mathrm{MM}$ patients, which is completely characterized for the major molecular alterations, i.e. chromosomal translocations involving the immunoglobulin heavy chain (IGH) locus, hyperdiploid (HD) status, deletions of 13q, 17p13, and gain of 1q. As shown in Figure 3 and Supplementary Table S2, among the 10 most differentially expressed lncRNAs in HD versus non HD (NHD) patients, we found nine upregulated lncRNAs that are all pseudogenes related to ribosomal protein coding genes; for these lncRNA-parental gene couples a highly positive correlation of their expression levels was found (Figure 3). Notably, in MM characterized by $t(11 ; 14)$ translocation the analysis unraveled the upregulation of lnc-LRRC47-1 and lnc-SEL1L3-6, and the downregulation of lnc-MC2R-2 and lnc-SPRYD7-2, the latter of which may have 25 putative transcripts, eight of which corresponding to DLEU2. Patients carrying $\mathrm{t}(4 ; 14)$ translocation upregulated lnc-WHSC2-2, a pseudogene located in the intron 19 of MMSET gene, which encodes the histone methyltransferase deregulated as the result of $\mathrm{t}(4 ; 14)$ translocation. Finally, MM samples with either the $\mathrm{t}(14 ; 16)$ or $\mathrm{t}(14 ; 20)$ translocations upregulated lncLIPG-3, the host gene of SCARNA17, and lnc-JAM2-2, which overlaps the MIR155HG locus.

Considering MM samples with 1q gain lesion, they specifically upregulated 7 lncRNAs all of which are located in the amplified chromosomal region and include lnc-SERPINC1-1 (GAS5) and lnc-CD46-4 that maps antisense to miR-102 gene. Patients with del13 significantly deregulated 14 lncRNAs, five of which reside on 13 q14 region. It is worth mentioning the downregulation of lnc-SPRYD7-1, also known as DLEU2, whose expression significantly correlated with that of miR-15a and miR-16-1 located in the same region (Supplementary Figure S3). MM patients with del17 upregulated 3 lncRNAs (Table 3).

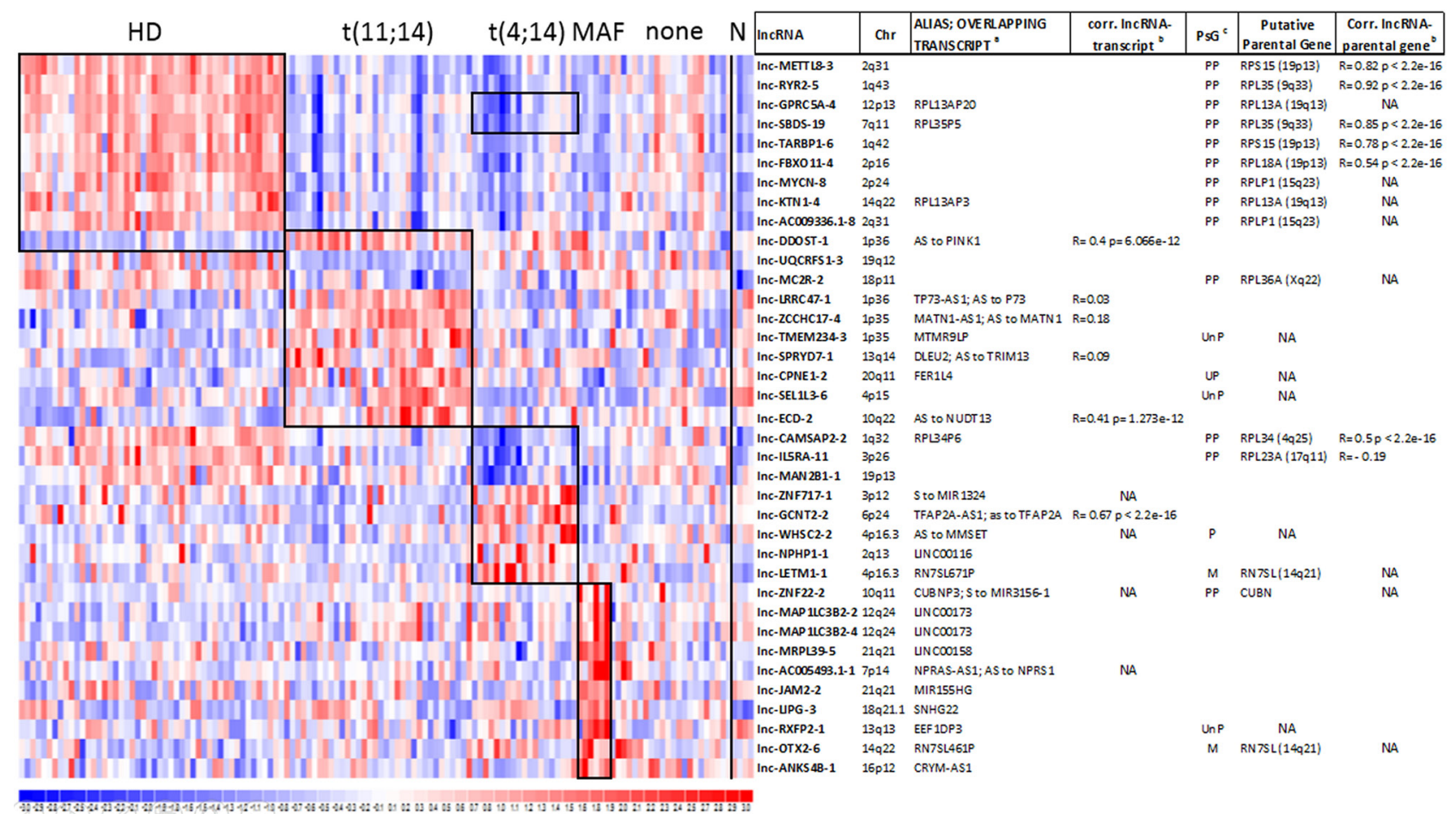

Figure 3: Identification of IncRNA signatures characterizing distinct MM genetic subgroups. Heatmap of the differentially expressed lncRNAs in 129 MM patients stratified into the five molecular groups as specified in methods; black boxes indicate the ten most differentially expressed lncRNAs resulting from the supervised analysis comparing each subgroup versus the rest of the dataset. LncRNA expression levels in normal plasma cells are shown on the right. For each lncRNA, information about chromosomal localization (Chr), alias name, transcripts overlapping in sense or antisense (indicated as S or AS, respectively) direction, and the Pearson's correlation coefficient between lncRNA and the overlapping transcript (when detected by the array) are indicated. For lncRNAs/pseudogenes, the last column reports the putative parental gene and the Pearson's correlation coefficient between lncRNA and the parental gene expression

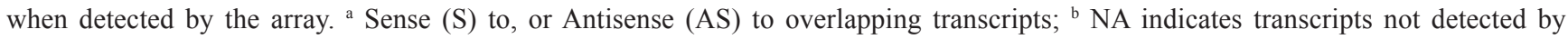
the array; ${ }^{c}$ PsG =pseudogene; Ensembl type: $\mathrm{PP}=$ Processed Pseudogene; $\mathrm{UP}=$ Unitary Pseudogene; UnP= Unprocessed Pseudogene; $\mathrm{M}=$ miscellaneous RNA. 
Table 3: LncRNAs significantly deregulated in MM patients with del13, del17 or 1q gain

\begin{tabular}{|c|c|c|c|c|c|c|c|c|}
\hline IncRNA & हृ & 등 & छี & $\begin{array}{c}\text { ALIAS; } \\
\text { OVERLAPPING } \\
\text { TRANSCRIPT }^{a}\end{array}$ & $\begin{array}{c}\text { Corr. } \\
\text { IncRNA- } \\
\text { overlapping } \\
\text { transcript }^{\mathrm{b}}\end{array}$ & $\mathbf{P s G}^{\mathrm{c}}$ & $\begin{array}{c}\text { Putative } \\
\text { Parental } \\
\text { Gene }\end{array}$ & $\begin{array}{l}\text { Corr. } \\
\text { IncRNA- } \\
\text { parental } \\
\text { gene }^{\text {b }}\end{array}$ \\
\hline \multicolumn{9}{|c|}{ del17 } \\
\hline MEF2C-2 & 4.98 & 2.26 & $5 q 14$ & $\begin{array}{l}\text { LINC00461; S to } \\
\text { MIR9-2 }\end{array}$ & NA & & & \\
\hline TRPV4-1 & 4.04 & 2.16 & $12 q 24$ & $\begin{array}{l}\text { FAM222A-AS1; AS } \\
\text { to FAM222A }\end{array}$ & $R=-0.07$ & & & \\
\hline ROPN1B-7 & 3.62 & 1.60 & $3 q 21$ & FAM86JP & & $\mathrm{PP}$ & $\begin{array}{l}\text { FAM86C1 } \\
(11 \mathrm{q} 13)\end{array}$ & $\mathrm{R}=0.22$ \\
\hline \multicolumn{9}{|c|}{ 1q gain } \\
\hline TOR1AIP2-5 & 3.81 & 1.48 & $1 \mathrm{q} 25$ & & & & & \\
\hline ANGPTL1-3 & 3.78 & 1.38 & $1 \mathrm{q} 25$ & AS to RALGPS2 & $\begin{array}{c}\mathrm{R}=0.3 \\
\mathrm{p}=3.8 \mathrm{e}-08\end{array}$ & & & \\
\hline CD46-4 & 3.43 & 1.30 & $1 \mathrm{q} 32$ & AS to miR-102 & NA & & & \\
\hline SERPINC1-1 & 3.24 & 1.38 & $1 q 25$ & $\begin{array}{l}\text { GAS5; AS to } \\
\text { ZBTB37 }\end{array}$ & $\begin{array}{c}\mathrm{R}=0.29 \\
\mathrm{p}=1.1 \mathrm{e}-06\end{array}$ & & & \\
\hline HNRNPU-1 & 3.12 & 1.48 & $1 q 44$ & $\begin{array}{l}\text { HNRNPU-AS1; AS to } \\
\text { COX20 }\end{array}$ & $\mathrm{R}=0.18$ & & & \\
\hline TRAF5-1 & 3.11 & 1.35 & $1 \mathrm{q} 32$ & LINC00467 & & & & \\
\hline ANKRD36BP1-1 & 2.90 & 1.30 & $1 \mathrm{q} 24$ & ANKRD36BP1 & & & & \\
\hline \multicolumn{9}{|c|}{ del13 } \\
\hline NEK3-1 & -4.07 & 0.70 & $13 q 14$ & MRPS31P5 & & UnP & NA & \\
\hline FAM60A-6 & -3.95 & 0.68 & $12 \mathrm{p} 11$ & $\mathrm{~S}$ to OVOS2 & NA & & & \\
\hline FAM70B-1 & -3.67 & 0.79 & $13 q 14$ & $\begin{array}{c}\text { GAS6-AS1; AS to } \\
\text { GAS6 }\end{array}$ & $\mathrm{R}=-0.28$ & & & \\
\hline CYorf15A.1-2 & -3.30 & 0.39 & Yq11 & TXLNG2P & & UnP & $\begin{array}{l}\text { TXLNG } \\
(\mathrm{Xp} 22)\end{array}$ & $\mathrm{R}=-0.07$ \\
\hline MTRNR2L1-4 & -3.28 & 0.85 & $17 \mathrm{p} 11$ & & & PP & MT-ND2 & NA \\
\hline ZFYVE1-4 & -3.03 & 0.87 & $14 \mathrm{q} 24$ & RN7SL586P & & M & $\begin{array}{l}\text { RN7SL } \\
(14 q 21)\end{array}$ & NA \\
\hline RNASEH1-7 & -2.96 & 0.81 & $2 \mathrm{p} 25$ & $\begin{array}{l}\text { TMSB4XP2; AS to } \\
\text { COLEC11 }\end{array}$ & $\mathrm{R}=0.19$ & UnP & $\begin{array}{c}\text { TMSB4X } \\
(\mathrm{Xp} 22)\end{array}$ & NA \\
\hline KDM5D-3 & -2.91 & 0.66 & Yq11 & & & & & \\
\hline SLC7A1-1 & -2.81 & 0.82 & $13 q 12$ & $\begin{array}{c}\text { MTUS2-AS1; AS to } \\
\text { MTUS2 }\end{array}$ & $\mathrm{R}=0.19$ & & & \\
\hline SPRYD7-1 & -2.70 & 0.72 & $13 q 14$ & $\begin{array}{l}\text { DLEU2; AS to } \\
\text { TRIM13 }\end{array}$ & $\mathrm{R}=0.09$ & & & \\
\hline RCOR3-1 & -2.67 & 0.87 & $1 \mathrm{q} 32$ & & & PP & $\begin{array}{l}\text { RPS25 } \\
(11 q 23)\end{array}$ & NA \\
\hline BACH1-2 & -2.67 & 0.84 & $21 \mathrm{q} 21$ & $\begin{array}{l}\text { GRIK1-AS1, AS to } \\
\text { GRIK1 }\end{array}$ & $\mathrm{R}=0.18$ & & & \\
\hline CLYBL-1 & -2.65 & 0.76 & $13 q 32$ & $\mathrm{~S}$ to CLYBL & $\mathrm{R}=0.24$ & & & \\
\hline POM121L2-2 & 3.77 & 1.80 & $6 \mathrm{p} 22$ & ZNF204P & & PP & NA & \\
\hline
\end{tabular}

${ }^{a}$ Sense (S) to, or Antisense (AS) to overlapping transcripts; ${ }^{b}$ Pearson correlation coefficient; NA =not available (not detected by the array); ${ }^{\mathrm{c}} \mathrm{PsG}=$ Pseudogene; Ensembl type: $\mathrm{PP}=$ Processed Pseudogene; UP= Unitary Pseudogene; UnP= Unprocessed Pseudogene; $\mathrm{M}=$ miscellaneous RNA. 
Table 4: Genes whose expression level highly correlate with that of IncRNAs resulting from SAM analyses

\begin{tabular}{|c|c|c|c|c|c|c|c|c|c|}
\hline $\operatorname{lncRNA}$ & Z & 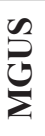 & $\sum_{\infty}^{\infty}$ & $\sum$ & $\underset{\varepsilon}{2}$ & Chr & ALIAS & $\begin{array}{l}\text { HIGHLY CORRELATED } \\
\text { GENE }\end{array}$ & $\begin{array}{l}\text { Corr. } \\
\text { IncRNA- } \\
\text { gene }^{\mathrm{a}}\end{array}$ \\
\hline C14orf49-4 & $\uparrow$ & $\downarrow$ & & & & $14 q 32$ & SNHG10 & RPL18A (19p13) & 0.907 \\
\hline RYR2-5 & $\downarrow$ & & $\uparrow$ & $\uparrow$ & & $1 q 43$ & RPL35P1 & RPL35 (9q33) & 0.918 \\
\hline TAMM41-3 & $\uparrow$ & $\downarrow$ & & & & $3 \mathrm{p} 25$ & RN7SL147P & $\begin{array}{l}\text { OCEL1 (occludin/ELL } \\
\text { domain containing } 1 ; 19 \mathrm{p} 13 \text { ) }\end{array}$ & 0.905 \\
\hline \multirow[t]{2}{*}{ CYorf15A.1-2 } & $\uparrow$ & & & & $\downarrow$ & Yq11 & $\begin{array}{l}\text { Taxilin } \\
\text { gamma 2, } \\
\text { pseudogene }\end{array}$ & $\begin{array}{l}\text { KDM5D (LYSINE- } \\
\text { SPECIFIC } \\
\text { DEMETHYLASE 5D; } \\
\text { Yq11) }\end{array}$ & 0.943 \\
\hline & & & & & & & & $\begin{array}{l}\text { USP9Y (UBIQUITIN- } \\
\text { SPECIFIC PROTEASE 9; } \\
\text { Yq11) }\end{array}$ & 0.910 \\
\hline \multirow[t]{2}{*}{ HSFY2-10 } & $\uparrow$ & & $\downarrow$ & $\downarrow$ & $\downarrow$ & Yq11 & CD24P4 & $\begin{array}{l}\text { BPI (BACTERICIDAL } \\
\text { PERMEABILITY- } \\
\text { INCREASING PROTEIN; } \\
\text { 20q11) }\end{array}$ & 0.914 \\
\hline & & & & & & & & $\begin{array}{l}\text { CEACAM8 } \\
\text { (CARCINOEMBRYONIC } \\
\text { ANTIGEN-RELATED } \\
\text { CELL ADHESION } \\
\text { MOLECULE 8; 19q13) }\end{array}$ & 0.923 \\
\hline
\end{tabular}

Arrows indicate lncRNA expression, upregulation $(\uparrow)$ or downregulation $(\downarrow)$, in the corresponding group. Chromosomal localization (Chr), alias name are indicated.

${ }^{a}$ Pearson correlation coefficient

\section{Correlation between IncRNA and gene expression levels}

Based on expression levels analysis, we evaluated for each of the differentially expressed lncRNA reported in Tables 1-3: i) the correlation with annotated transcripts that mapped sense or antisense to it, i.e. a potential in cis regulation; and ii) the relationship between lncRNAs/ pseudogenes and matching parental genes. The results, reported in Tables 1-3, pointed out that pseudogenes are often co-expressed at variable levels with their putative parental gene. Furthermore, to gain evidences of lncRNAs that may potentially act on gene expression, we extended this correlation analysis to all the 17788 transcripts unambiguously detectable by the arrays. To be confident of identifying lncRNA-gene real interaction, we focused on correlation coefficient $>0.9$, and found five IncRNAs that highly correlated with one or more genes (Table 4), all of them modulated between normal and pathological samples. Among these, besides lnc-cYorf15A.1-2 and KDM5D gene that map $\sim 112 \mathrm{~kb}$ apart on chromosome $\mathrm{Y}$, the other four lncRNAs correlated with genes located on different chromosomes, suggesting in trans interplay for such couples.

\section{Quantitative RT-PCR validation of differentially expressed IncRNAs}

The expression levels of six relevant lncRNAs found differentially expressed in our analyses were investigated by qRT-PCR performed in $60 \mathrm{MM}$ samples for whom RNA material was available. Specifically, we validated the expression levels of the well-known MALAT1, GAS5, and DLEU2 IncRNAs. Furthermore PCR results confirmed the significant upregulation of GAS5 in samples with 1q gain lesion, and the downregulation of DLEU2 in patients carrying del13 (Figure 4A-4B). Likewise, we validated the expression of lnc-ANGPTL1-3, lnc-SENP5-4, and lncLRRC47-1, found progressively deregulated in association with more aggressive forms of the disease (Figure 4C).

\section{Functional annotation of MALAT1 signature in MM}

Although we found several lncRNAs deregulated in the different form of PC dyscrasia, only a very few number have been functionally validated in biological and disease processes. Among these, we found MALAT1, the 
A

SPRYD7-1 (DLEU2 13q14)

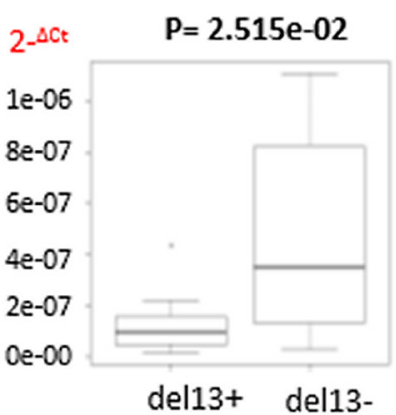

C

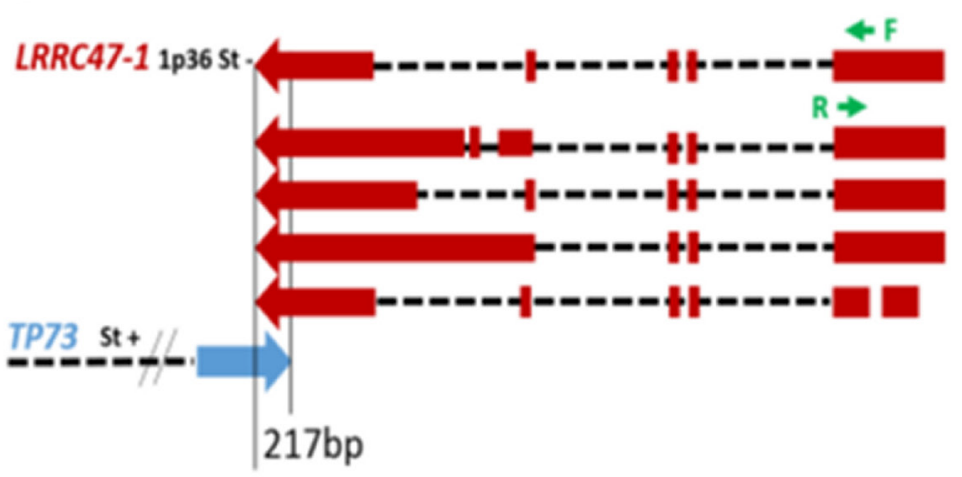

B

SERPINC1-1 (GAS5 1q25)

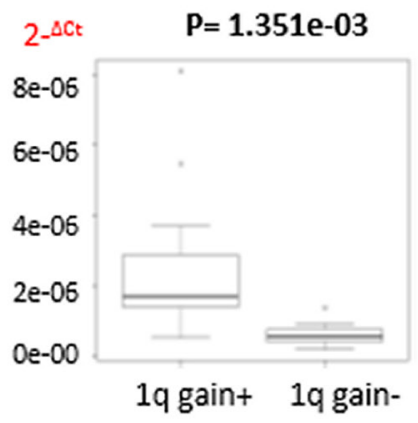

SCYL1-1 (MALAT1 11q13)

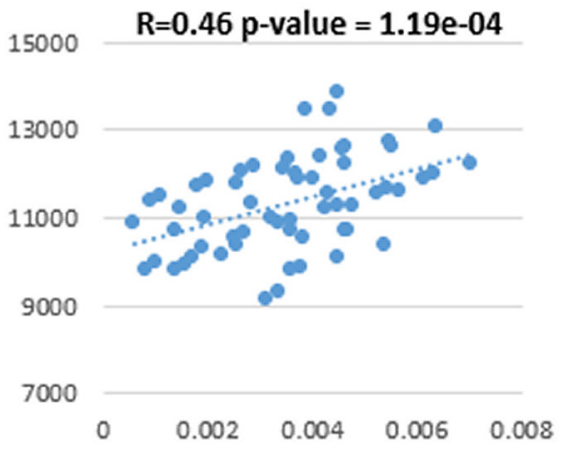

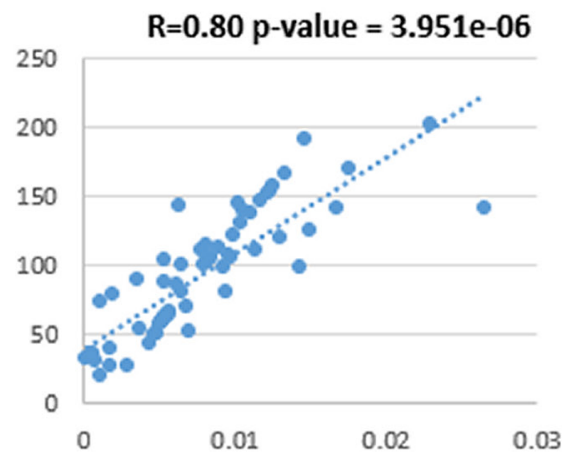

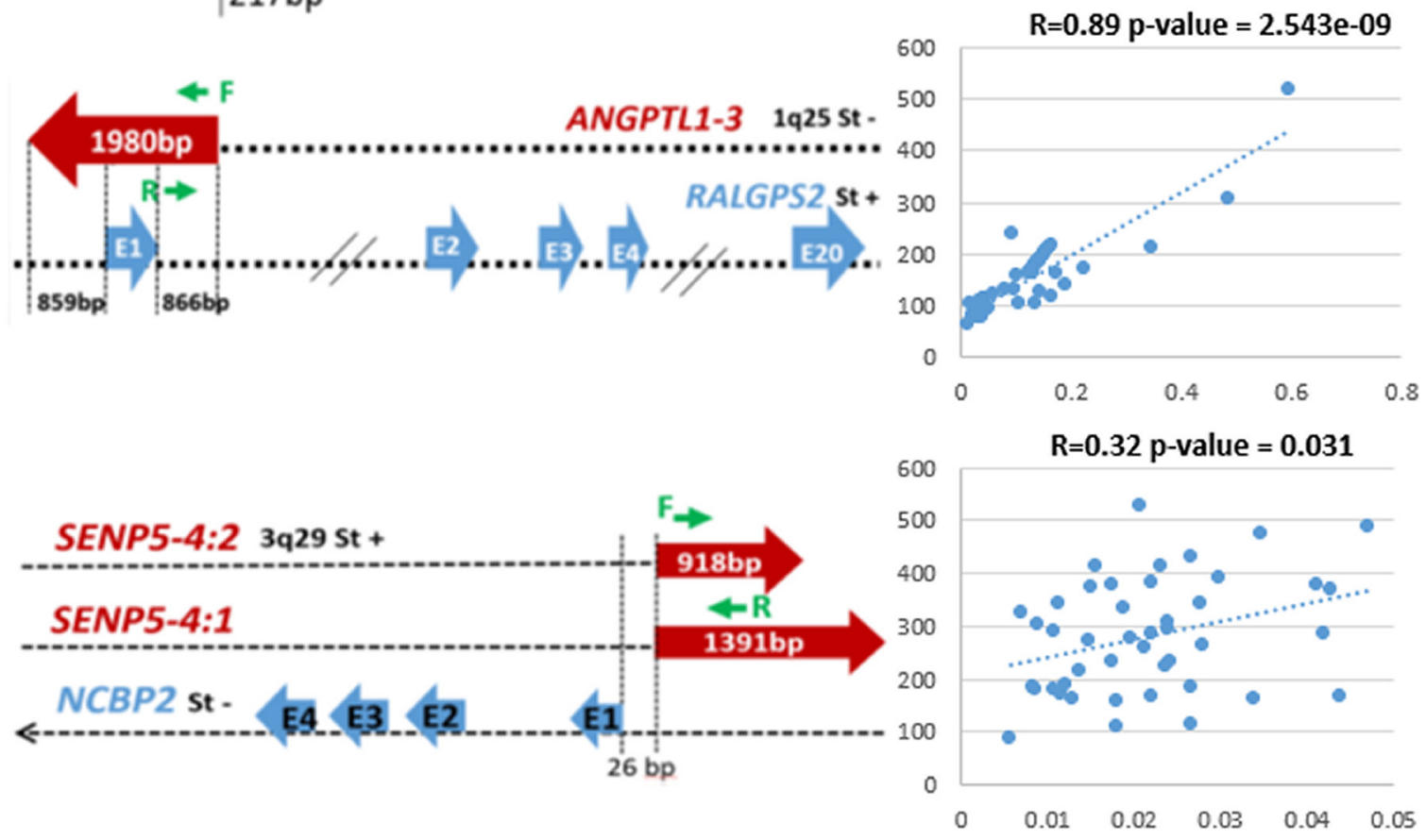

Figure 4: Quantitative RT-PCR validation of IncRNA expression in 60 MM cases. A. Box plots of lnc-SPRYD7-1 and lncSERPINC1-1 expression show a significant correlation with group membership based on Wilcoxon rank-sum tests ( $p$-values are shown above each panel). The expression levels are represented as $2^{-\Delta C t}$. B. MALAT1 expression validation; Pearson's correlation coefficient was calculated between GEP data and quantitative RT-PCR results expressed as $2^{-\Delta \mathrm{Ct}}$. C. Genomic map of $\operatorname{lncRNAs}$ (red) and antisense genes (blue) with custom primers (green) localization. Sense or antisense chromosomal position (St+ or St-, respectively) are specified. For lnc-LRRC47-1, the scheme was limited to longer transcripts. Pearson's correlation coefficient was calculated between GEP data and quantitative RT-PCR results expressed as $2^{-\Delta C t}$. 
abundant nuclear-retained lncRNA found overexpressed in several cancers associated with high proliferation and metastasis, although the underlying mechanism(s) behind this deregulation and its significance in tumorigenesis is still poorly understood. Our data demonstrates a significant overexpression of MALAT1 in tumor samples compared to healthy controls (Figure 5A). To gain insight into the role of MALAT1 in MM, we focused on the proprietary MM group (due to the absence of any bias related to sample molecular characteristics) and looked for the gene expression signature associated with MALAT1 expression. Specifically, we ranked patients in four classes based on MALAT1 expression level. Next, by comparing the less (I quartile) with the most (IV quartile) expressing patients, we found 518 genes differentially expressed between the two groups that appeared to be gradually modulated in the four quartiles (Figure 5B and Supplementary Table S3). Functional enrichment analysis of the 518 deregulated genes using ToppGene evidenced 88 significantly enriched pathways, many of which attributable to mRNA maturation, proteasome degradation, cell cycle processes, and p53-dependent G1/S DNA damage checkpoint (Supplementary Table S4). In addition, Gene Set Enrichment Analysis (GSEA) was used to identify a priori defined sets of genes showing concordant modulation between patients with low and high MALAT1 expression level. Notably, GSEA analysis identified 13 gene sets (Figure 5C and Supplementary Table S5) among which the pathway associated with p53-mediated DNA damage response was highly enriched in MM group expressing less MALAT1 (Figure 5D).

\section{DISCUSSION}

LncRNAs are an emerging field of investigation as they are suggested to regulate key biological processes, including cellular proliferation and differentiation, and their aberrant expression being strongly associated with cancer [25].

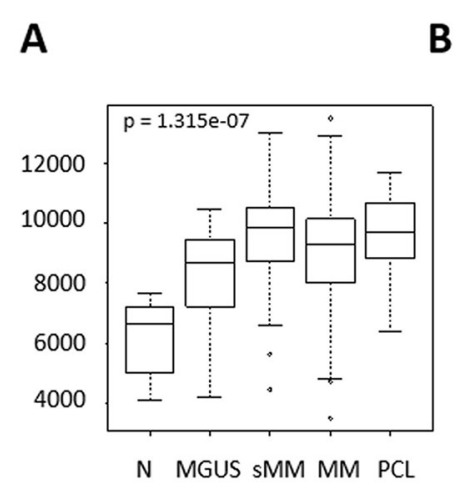

B

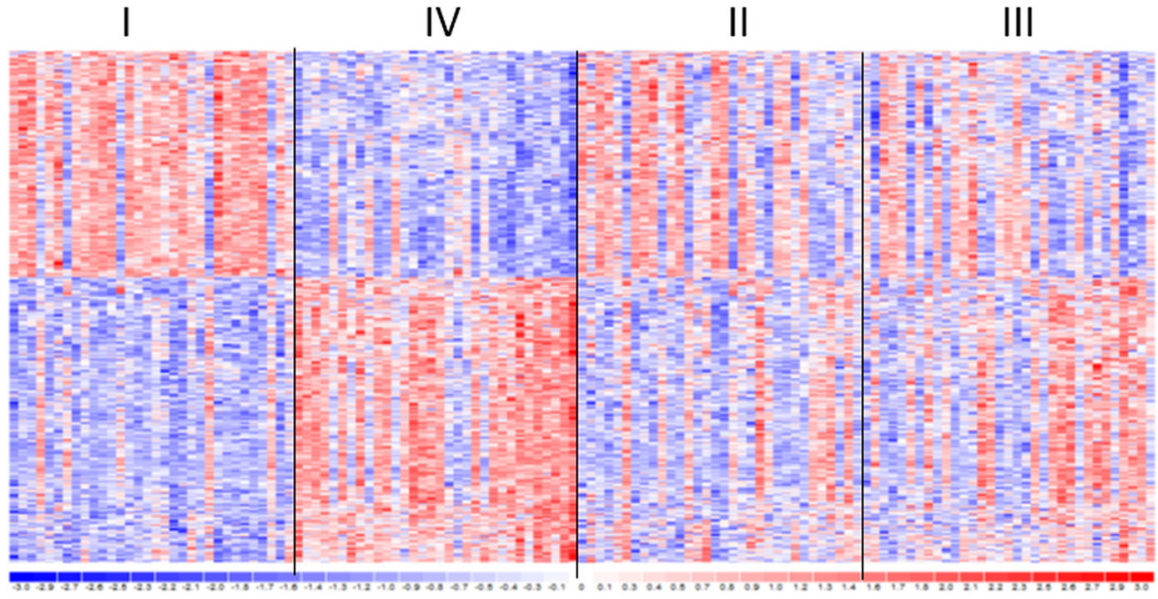

C

\begin{tabular}{lcc}
\hline \multicolumn{1}{c}{ Gene Set } & NES & NOM p-Val \\
\hline REACTOME_CASPASE_MEDIATED_CLEAVAGE_OF_CYTOSKELETAL_PROTEINS & 1.8397 & 0.0000 \\
BIOPOLYMER_BIOSYNTHETIC_PROCESS & 1.7961 & 0.0040 \\
ATP_DEPENDENT_DNA_HELICASE_ACTIVITY & 1.7101 & 0.0021 \\
SMOOTH_MUSCLE_CONTRACTION_GO_OOO6939 & 1.7078 & 0.0083 \\
BOYAULT_LIVER_CANCER_SUBCLASS_G6_DN & 1.6905 & 0.0042 \\
KOBAYASHIEGFR_SIGNALING_6HR_UP & 1.5956 & 0.0021 \\
DRUG_BINDING & 1.5939 & 0.0079 \\
KERLEY_RESPONSE_TO_CISPLATIN_DN & 1.5391 & 0.0099 \\
ATP_DEPENDENT_HELICASE_ACTIVITY & -1.7369 & 0.0097 \\
REACTOME_NOREPINEPHRINE_NEUROTRANSMITTER_RELEASE_CYCLE & -1.6592 & 0.0058 \\
NADERI_BREAST_CANCER_PROGNOSIS_DN & -1.6542 & 0.0058 \\
REACTOME_DOPAMINE_NEUROTRANSMITTER_RELEASE_CYCLE & -1.6171 & 0.0098 \\
DNA_DAMAGE_RESPONSESIGNAL_TRANSDUCTION_RESULTING_IN_INDUCTION_OF_APOPTOSIS & -1.5768 & 0.0059 \\
\hline
\end{tabular}

D

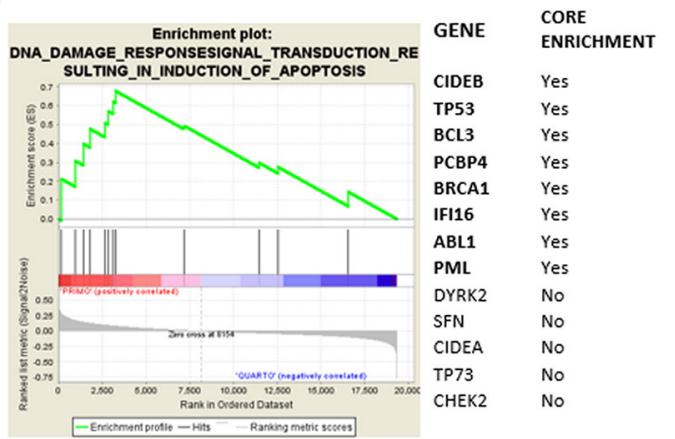

Figure 5: MALAT1 expression deregulation in MM patients. A. Box plot of MALAT1 expression level in 9 N, 20 MGUS, 33 SMM, 170 MM, and 36 PCL samples, as detected by GEP; $p$-value was calculated by Kruskal-Wallis rank sum test. B. Heatmap of the 518 differentially expressed lncRNAs identified by comparing the first $v s$ fourth quartile of $129 \mathrm{MM}$ patients stratified into four groups based on MALAT1 expression level. C. Gene sets significantly up- and down-regulated in MALAT1 quartile IV versus I. Gene sets were selected on nominal p-value $<0.01$ and are ordered according to NES value. D. Enrichment plot of "DNA damage response signal transduction resulting in induction of apoptosis" gene set detected by GSEA. The green curves show the enrichment score and reflect the degree to which each gene (black vertical lines) is represented at the bottom of the ranked gene list. Genes contributing to the core enrichment in the gene set are indicated in bold. 
To the best of our knowledge, this is the first report describing the global expression profiles of lncRNAs in a large cohort of samples representing all the major different forms of plasma cell dyscrasias. Our study identified 31 lncRNAs that were specifically deregulated in this pathology compared to normal bone marrow PC controls (Table 1). In particular, we found six lncRNAs deregulated in all the different clinical forms of the disease, whereas 18 others appear associated with more advanced phases of the disease, as their expression in MGUS samples does not differ significantly from normal controls. A very notable example among lncRNAs found upregulated in pathological samples, is MALAT1 that has been widely reported to be upregulated in a large variety of solid tumors in association with progression and cancer metastasis [21]. It may be surprising that five lncRNAs are significantly downregulated in MGUS, SMM and PCL groups but not in MM one. This finding may be explained by the great heterogeneity of the MM patient. In agreement with this hypothesis, three out of the five lncRNAs, specifically lnc-SNURF-1, lnc-SNURF-3, and lnc-SEL1L3-6, were significantly deregulated in distinct MM subgroups (Supplementary Table S2).

Notably, we identified 21 lncRNAs whose expression was progressively deregulated in association with the increased aggressiveness of the disease (Table 2). Among these, the expression levels of lnc-SENP5-4, lnc-CPSF2-2, and lnc-LRRC47-1 were found to be significantly different in a cohort of $19 \mathrm{MM}$ patients at diagnosis compared to the corresponding relapse/PCL progressed phases (Supplementary Figure S2), a finding that further supports the previous evidence. In addition, a recent study investigating lnc-LRRC47-1 in MM, confirmed its progressive downregulation from normal PCs to MGUS and symptomatic disease, which appeared independent from the methylation status of its promoter [26]. Thus, it is conceivable that these lncRNAs may have a role in the progression of the disease. Furthermore, disease progression is associated with the upregulation of GAS5 and lnc-ANGPTL1-3, both located at 1q25; this finding is in line with the GEP-model for high-risk MM that is enriched in overexpressed genes mapping to chromosome 1q [27]. Certainly the role of GAS5 in MM deserves further studies considering that almost all the existent literature describes GAS5 down-regulation in different cancers, above all solid tumors; according to existing data, GAS5 both inhibits the proliferation and promotes the apoptosis of multiple cell types, consistent with a tumor suppressor role (reviewed in [28]). To date, GAS5 overexpression in tumor has been reported only in mesothelioma [29], where the Authors hypothesized, as a possible explanation of the discrepancy, that high GAS5 expression in that cellular milieu does not only control cell cycle but also act as a natural sponge for miRNAs. GAS5 has been demonstrated as host gene for snoRNA molecules that traditionally function as guide for the post-transcriptional modification of ribosomal and some spliceosomal RNAs and are supposed to play a role in alternative splicing processes [30]. According to this, we may hypothesize that in PC dyscrasia GAS5 deregulation affects processes that represent oncogenic mechanisms in MM.

In the context of MM, we demonstrated that deregulated patterns of lncRNAs expression are associated with distinct molecular subtypes, as it has long been commonly accepted for mRNAs, miRNAs, and snoRNAs [30-33]. Despite the fact that the mechanism and the function of lncRNAs, as well as the consequences of their deregulation remain to be fully clarified, the transcriptional lncRNA signature of some MM subgroups may not be surprising. In fact, the most upregulated lncRNAs in HD tumors are all pseudogenes of parental ribosomal protein coding genes, with which they are co-expressed (Figure 3). These findings are in line with the global up-regulation of the translational machinery, including genes involved in protein biosynthesis, characterizing this molecular group [34]. Based on the suggestion that the presence of pseudogenes may serve to modulate the activity of their parental gene $[35,36]$, it is feasible that these lncRNAs/ pseudogenes may act as indirect post-transcriptional regulators decoying ncRNA, in particular miRNAs that target the parental gene [36]. Very interesting is also the Inc-JAM2-2, found to be upregulated in MM patients with MAF deregulation; this lncRNA overlaps MIR155HG gene, i.e. the host gene of the miR-155 found also upregulated in translocated MAF samples [31]. Considering $\mathrm{MM}$ patients carrying $\mathrm{t}(4 ; 14)$, the upregulation of lnc-WHSC2-2 that maps intronic and antisense to the translocation target gene MMSET, is relevant. Notably, the consistent deregulation of lncWHSC2-2 in translocated MM resembles that of the snoRNA ACA11 that maps in the adjacent 3' intron of MMSET on the sense strand [30,37]. It is conceivable that, as reported for ACA11 [37], lnc-WHSC2-2 may be a critical target of the $\mathrm{t}(4 ; 14)$ translocation in $\mathrm{MM}$ with a specific oncogenic role. Finally, the 1q-gain MM group showed significantly upregulated seven lncRNAs all located at 1q region, suggesting that gene dose effect may also be a mechanism behind lncRNAs deregulation.

Although every resultant IncRNA from our study warrants further investigation to elucidate its possible biological role, some of them are suggestive of a pathological function merely by relying on their genomic position. In particular, the overexpression of lncPTPDC1-7, lnc-USP25-2, and lnc-MON2-2 that overlap host genes for members of the let-7 family may somehow be related to this miRNA downregulation often detected in cancer [38]. Other lncRNAs lie in proximity of genes already known in cancer and thus potentially involved in their regulation, for example lnc-LRRC47-1, which may regulate the tumor suppressor TP73 gene [39]. 
At present, there is strong evidence of a major role for lncRNAs in tumorigenesis based on several hundred lncRNAs already identified as being aberrantly expressed in cancer [25]. However, only a few of these lncRNAs have been functionally validated in normal and pathological biologic processes. Among these, MALAT1 was reported to regulate cellular proliferation by modulating the expression and/or pre-mRNA processing of cell cycle-regulated transcription factors; moreover, transient overexpression of MALAT1 enhanced cellular proliferation in cell lines and tumor formation in nude mice, while its depletion in tumor cells reduced tumorigenicity $[40,41]$. In line with these findings, our data evidenced a significant overexpression of MALAT1 in tumor samples compared to healthy controls. Interestingly, functional annotation of the MALAT1 signature in MM identified enriched pathways linked to mRNA maturation, proteasome degradation, cell cycle processes, and p53dependent G1/S DNA damage checkpoint. In addition, GSEA results showed that the pathway associated with p53-mediated DNA damage response was highly enriched in MM group less expressing MALAT1. This evidence suggests that p53 may be an important effector of MALAT1 function in PCs as it has been described in fibroblast [42].

In conclusion, our study reported many lncRNAs deregulated in the different forms of plasma cell dyscrasia. The challenge now and in the future is to identify the truly oncogenic and functionally relevant lncRNAs.

\section{MATERIALS AND METHODS}

\section{Samples}

The study was performed in a cohort of 268 patients included in two different datasets, and representative of all the major forms of plasma cell dyscrasia. Specifically, the proprietary dataset publicly available at the NCBI Gene Expression Omnibus repository (accession \#GSE66293) included 4 normal controls (Voden, Medical Instruments IT), $129 \mathrm{MM}, 24 \mathrm{pPCL}$, and 12 sPCL patients; the other [43] included 5 normal controls, 20 MGUS, 33 SMM, and 41 MM patients, whose expression data were publicly available (accession \#GSE47552). With the exception of sPCL, the cohort consists of newly-diagnosed patients. PCs were purified from bone marrow samples using CD138 immunomagnetic microbeads (MidiMACS system, Miltenyi Biotec, Auburn, CA) and the purity of the positively selected PCs was $>90 \%$ in all cases.

The proprietary $129 \mathrm{MM}$ tumors employed for the study were representative of the major molecular characteristics of the disease. Samples were characterized for the presence of the most frequent chromosomal translocations and the ploidy status based on fluorescence in situ hybridization (FISH) evaluation criteria, as previously described [34]. Specifically, forty-eight showed a HD status; thirty-four samples were characterized by the $\mathrm{t}(11 ; 14)$ or $\mathrm{t}(6 ; 14)$ translocations; nineteen had the $\mathrm{t}(4 ; 14)$ translocation; six had either the $\mathrm{t}(14 ; 16)$ or $\mathrm{t}(14 ; 20)$ translocations; and twenty-two did not fall into any of the other groups. Deletions of 17p13,13q14 and gain of $1 \mathrm{q}$ were also evaluated by FISH.

\section{Expression profiling}

GEP of the 268 samples was generated using GeneChip ${ }^{\circledR}$ Gene 1.0 ST Array (Affymetrix Inc., Santa Clara, CA) as previously described [33] (Supplementary Methods). Then, we applied a custom pipeline (Figure 1) to detect specific lncRNAs. Log2-transformed expression values were extracted from CEL files and normalized using RMA procedure in Expression Console Software (Affymetrix, Inc.) at the probe cluster ID annotation level. Cross-hybridization probes were filtered-out. To avoid potential biases due to the mixing of two different datasets, a quality control of the array datasets was performed. In particular, before proceeding, to prevent the inclusion of low-quality or non-reproducible data, normalized unscaled standard error (NUSE) and relative log-expression (RLE) distributions were generated in aroma.affymetrix package for all samples (samples would be removed if the 25th or the 75th percentile of NUSE and RLE exceeded the value of \pm 1.05 or \pm 0.5 , respectively; Supplementary Figure S4A-B). Then, we combined annotated probes with both Ensembl transcripts (GRCh37/hg19 assembly) and lncRNAs from LNCipedia repository (http://www. lncipedia.org/), based on the chromosome localization of the target sequence identified by each probe; afterwards, we considered only probes univocally referable to lncRNA transcripts (i.e. that do not overlap with Ensembl transcripts). To summarize probes related to each lncRNA, we considered their median expression value. Finally, we added a further step of batch adjustment using the sva function in the homonymous package of the R software, to avoid any possible cohort-specific bias due to the merging of different datasets. Through this annotation pipeline, we selectively detected 1852 lncRNAs from LNCipedia database.

The principal component analysis (PCA) of the samples was performed by singular value decomposition of the considered data expression matrix using the prcomp function in the stats package, and the results were visualized using the plot $3 d$ function in the $r g l$ package of the R software (Supplementary Figure S4C). Supervised analyses were carried out using the Significant Analysis of Microarrays software version 5.00 [44] using the web application provided in the shiny package of the $\mathrm{R}$ software (https://github.com/MikeJSeo/SAM). The cutoff point for statistical significance (at a $q$-value 0 ) was determined by tuning the $\Delta$ parameter on the false discovery rate and controlling the q-value of the selected probes. Hierarchical agglomerative clustering 
of patients based on the most significant probesets found was performed adopting Pearson's correlation and average as distance and linkage methods, respectively. DNA-Chip Analyzer software (dChip) [45] was used to perform clustering and graphically represent it. The list of differentially expressed genes was submitted to the ToppGene Suite portal (http://toppgene.cchmc.org) for functional enrichment analysis using the ToppFun application [46]. Microarray data were globally analyzed by Gene Set Enrichment Analysis (GSEA) [47]. MiRNA expression data was available for 125 samples out of our series (GSE70254 and GSE73452) generated using GeneChip ${ }^{\circledR}$ miRNA 3.0 Array (Affymetrix Inc., Santa Clara, CA) as previously described [48].

\section{Quantitative real-rime PCR (qRT-PCR)}

For qRT-PCR, 100 ng of total RNA underwent reverse transcription using random primer mix and reagents from INVITROGEN (Life Technologies, Foster City, CA). Real-time PCR was performed in triplicate using TaqMan ${ }^{\circledR}$ Non-coding RNA Assays (Hs00273907 s1, Hs03671981 s1, and Hs00863925 m1 for MALAT1, GAS5, and DLEU, respectively) together with the TaqMan ${ }^{\circledR}$ Fast Universal PCR Master Mix on an Applied Biosystems 7900 Sequence Detection System. RNA samples were normalized based on the house-keeping $18 S$ rRNA. Real-time PCR to validate lnc-LRRC47-1, lnc-ANGPTL1-3, and lnc-SENP5-4 was performed using 10 ng of total RNA and SYBR ${ }^{\mathrm{TM}}$ Green master mixes with custom primers (Supplementary Table S6). RNA samples were normalized based on the GAPDH gene. The threshold cycle $\left(\mathrm{C}_{\mathrm{T}}\right)$ was defined as the fractional cycle number at which the fluorescence passes the fixed threshold. LncRNA expression was relatively quantified using the $2^{-\Delta \mathrm{Ct}}$ method (Applied Biosystems User Bulletin No. 2), and expressed as the relative quantity of target IncRNA normalized to the house-keeping $18 S$ rRNA.

\section{Statistical analysis}

Conventional statistical procedures were applied using standard packages of the $\mathrm{R}$ software (Kendall $\tau$ correlations and Wilcoxon rank-sum tests). The Jonckheere-Terpstra test function was used in the clinfun package to investigate the significance of the trend from normal donors through PCL cases in the expression levels of the selected lncRNA list generated on Gene 1.0 ST array. For robustness, to reduce biases that might be due to numerical imbalances within the groups (with the MM group being largely overrepresented) and to gain the most significant trends, an additional criterion was imposed that only steadily ascending or descending median values were allowed. In addition, a differential expression should be observed in at least 1 condition (Kruskal-Wallis tests using the appropriate function in the stat package in $\mathrm{R}$ software). The Benjamini and Hochberg correction was used to adjust significance of multiple tests (adjusted $P$ $<0.05$ ).

\section{CONFLICTS OF INTERESTS}

The authors declare no conflicts of interests.

\section{GRANT SUPPORT}

This work was financially supported by grants from: the AIRC Investigator Grant no.10136 (to AN), and the AIRC Special Program Molecular Clinical Oncology, 5 per mille no. 9980. K. Todoerti was supported in part by the Italian Health Minister, Finalized Research for Young Researchers, CUP Project E66110000230001.

\section{REFERENCES}

1. Kuehl WM, Bergsagel PL. Multiple myeloma: evolving genetic events and host interactions. Nat Rev Cancer. 2002; 2:175-187.

2. Kyle RA, Rajkumar SV. Monoclonal gammopathies of undetermined significance: a review. Immunol.Rev. 2003; 194:112-139.

3. Palumbo A, Anderson K. Multiple myeloma. N Engl J Med. 2011; 364:1046-1060.

4. Munshi NC, Anderson KC. New strategies in the treatment of multiple myeloma. Clin Cancer Res. 2013; 19:3337-3344.

5. Birney E, Stamatoyannopoulos JA, Dutta A, Guigo R, Gingeras TR, Margulies EH, Weng Z, Snyder M, Dermitzakis ET, Thurman RE, Kuehn MS, Taylor CM, Neph S, et al. Identification and analysis of functional elements in $1 \%$ of the human genome by the ENCODE pilot project. Nature. 2007; 447:799-816.

6. Djebali S, Davis CA, Merkel A, Dobin A, Lassmann T, Mortazavi A, Tanzer A, Lagarde J, Lin W, Schlesinger F, Xue C, Marinov GK, Khatun J, et al. Landscape of transcription in human cells. Nature. 2012; 489:101-108.

7. Fabian MR, Sonenberg N. The mechanics of miRNAmediated gene silencing: a look under the hood of miRISC. Nat Struct Mol Biol. 2012; 19:586-593.

8. Kong YW, Ferland-McCollough D, Jackson TJ, Bushell M. microRNAs in cancer management. Lancet Oncol. 2012; 13:e249-e258.

9. Amodio N, Di Martino MT, Neri A, Tagliaferri P, Tassone P. Non-coding RNA: a novel opportunity for the personalized treatment of multiple myeloma. Expert Opin Biol Ther. 2013; 13 Suppl 1:S125-S137.

10. Berindan-Neagoe I, Monroig PC, Pasculli B, Calin GA. MicroRNAome genome: a treasure for cancer diagnosis and therapy. CA Cancer J Clin. 2014; 64:311-336. 
11. Garzon R, Marcucci G, Croce CM. Targeting microRNAs in cancer: rationale, strategies and challenges. Nat Rev Drug Discov. 2010; 9:775-789.

12. Cech TR, Steitz JA. The noncoding RNA revolutiontrashing old rules to forge new ones. Cell. 2014; 157:77-94.

13. Milligan MJ, Lipovich L. Pseudogene-derived lncRNAs: emerging regulators of gene expression. Front Genet. 2014; 5:476.

14. Cabili MN, Trapnell C, Goff L, Koziol M, Tazon-Vega B, Regev A, Rinn JL. Integrative annotation of human large intergenic noncoding RNAs reveals global properties and specific subclasses. Genes Dev. 2011; 25:1915-1927.

15. Mercer TR, Mattick JS. Structure and function of long noncoding RNAs in epigenetic regulation. Nat Struct Mol Biol. 2013; 20:300-307.

16. Sun M, Kraus WL. From discovery to function: the expanding roles of long noncoding RNAs in physiology and disease. Endocr Rev. 2015; 36:25-64.

17. Ling H, Vincent K, Pichler M, Fodde R, Berindan-Neagoe I, Slack FJ, Calin GA. Junk DNA and the long noncoding RNA twist in cancer genetics. Oncogene. 2015; 34:5003-5011.

18. Yang G, Lu X, Yuan L. LncRNA: a link between RNA and cancer. Biochim.Biophys.Acta. 2014; 1839:1097-1109.

19. Volders PJ, Verheggen K, Menschaert G, Vandepoele K, Martens L, Vandesompele J, Mestdagh P. An update on LNCipedia: a database for annotated human lncRNA sequences. Nucleic Acids Res. 2015; 43:D174-D180.

20. Zhou M, Zhao H, Wang Z, Cheng L, Yang L, Shi H, Yang H, Sun J. Identification and validation of potential prognostic lncRNA biomarkers for predicting survival in patients with multiple myeloma. J Exp Clin Cancer Res. 2015; 34:102-115

21. Tripathi V, Ellis JD, Shen Z, Song DY, Pan Q, Watt AT, Freier SM, Bennett CF, Sharma A, Bubulya PA, Blencowe BJ, Prasanth SG, Prasanth KV. The nuclear-retained noncoding RNA MALAT1 regulates alternative splicing by modulating SR splicing factor phosphorylation. Mol Cell. 2010; 39:925-938.

22. Yang F, Yi F, Han X, Du Q, Liang Z. MALAT-1 interacts with hnRNP C in cell cycle regulation. FEBS Lett. 2013; 587:3175-3181.

23. Cho SF, Chang YC, Chang CS, Lin SF, Liu YC, Hsiao $\mathrm{HH}$, Chang JG, Liu TC. MALAT1 long non-coding RNA is overexpressed in multiple myeloma and may serve as a marker to predict disease progression. BMC Cancer. 2014; 14:809-817.

24. Isin M, Ozgur E, Cetin G, Erten N, Aktan M, Gezer U, Dalay N. Investigation of circulating lncRNAs in B-cell neoplasms. Clin Chim Acta. 2014; 431:255-259.

25. Brunner AL, Beck AH, Edris B, Sweeney RT, Zhu SX, Li R, Montgomery K, Varma S, Gilks T, Guo X, Foley JW, Witten DM, Giacomini CP et al. Transcriptional profiling of long non-coding RNAs and novel transcribed regions across a diverse panel of archived human cancers. Genome Biol. 2012; 13:R75-87.

26. Wong KY, Li Z, Zhang X, Leung GK, Chan GC, Chim CS. Epigenetic silencing of a long non-coding RNA KIAA0495 in multiple myeloma. Mol Cancer. 2015; 14:175-179.

27. Shaughnessy JD, Jr., Zhan F, Burington BE, Huang Y, Colla S, Hanamura I, Stewart JP, Kordsmeier B, Randolph C, Williams DR, Xiao Y, Xu H, Epstein J et al. A validated gene expression model of high-risk multiple myeloma is defined by deregulated expression of genes mapping to chromosome 1. Blood. 2007; 109:2276-2284.

28. Pickard MR, Williams GT. Molecular and Cellular Mechanisms of Action of Tumour Suppressor GAS5 LncRNA. Genes (Basel). 2015; 6:484-499.

29. Renganathan A, Kresoja-Rakic J, Echeverry N, Ziltener G, Vrugt B, Opitz I, Stahel RA, Felley-Bosco E. GAS5 long non-coding RNA in malignant pleural mesothelioma. Mol Cancer. 2014; 13:119-130.

30. Ronchetti D, Todoerti K, Tuana G, Agnelli L, Mosca L, Lionetti M, Fabris S, Colapietro P, Miozzo M, Ferrarini M, Tassone P, Neri A. The expression pattern of small nucleolar and small Cajal body-specific RNAs characterizes distinct molecular subtypes of multiple myeloma. Blood Cancer J. 2012; 2:e96-103.

31. Lionetti M, Biasiolo M, Agnelli L, Todoerti K, Mosca L, Fabris S, Sales G, Deliliers GL, Bicciato S, Lombardi L, Bortoluzzi S, Neri A. Identification of microRNA expression patterns and definition of a microRNA/mRNA regulatory network in distinct molecular groups of multiple myeloma. Blood. 2009; 114:e20-e26.

32. Mattioli M, Agnelli L, Fabris S, Baldini L, Morabito F, Bicciato S, Verdelli D, Intini D, Nobili L, Cro L, Pruneri G, Callea V, Stelitano C, et al. Gene expression profiling of plasma cell dyscrasias reveals molecular patterns associated with distinct IGH translocations in multiple myeloma. Oncogene. 2005; 24:2461-2473.

33. Todoerti K, Agnelli L, Fabris S, Lionetti M, Tuana G, Mosca L, Lombardi L, Grieco V, Bianchino G, D'Auria F, Statuto T, Mazzoccoli C, De LL, et al. Transcriptional characterization of a prospective series of primary plasma cell leukemia revealed signatures associated with tumor progression and poorer outcome. Clin Cancer Res. 2013; 19:3247-3258.

34. Agnelli L, Fabris S, Bicciato S, Basso D, Baldini L, Morabito F, Verdelli D, Todoerti K, Lambertenghi-Deliliers G, Lombardi L, Neri A. Upregulation of translational machinery and distinct genetic subgroups characterise hyperdiploidy in multiple myeloma. Br J Haematol. 2007; 136:565-573.

35. Gupta V, Warner JR. Ribosome-omics of the human ribosome. RNA. 2014; 20:1004-1013.

36. Muro EM, Mah N, Andrade-Navarro MA. Functional evidence of post-transcriptional regulation by pseudogenes. Biochimie. 2011; 93:1916-1921. 
37. Chu L, Su MY, Maggi LB, Jr., Lu L, Mullins C, Crosby S, Huang G, Chng WJ, Vij R, Tomasson MH. Multiple myeloma-associated chromosomal translocation activates orphan snoRNA ACA11 to suppress oxidative stress. J Clin Invest. 2012; 122:2793-2806.

38. Peter ME Let-7 and miR-200 microRNAs: guardians against pluripotency and cancer progression. Cell Cycle. 2009; 8:843-852.

39. Candi E, Agostini M, Melino G, Bernassola F. How the TP53 family proteins TP63 and TP73 contribute to tumorigenesis: regulators and effectors. Hum Mutat. 2014; 35:702-714.

40. Gutschner T, Hammerle M, Eissmann M, Hsu J, Kim Y, Hung G, Revenko A, Arun G, Stentrup M, Gross M, Zornig M, MacLeod AR, Spector DL, et al. The noncoding RNA MALAT1 is a critical regulator of the metastasis phenotype of lung cancer cells. Cancer Res. 2013; 73:1180-1189.

41. Li L, Feng T, Lian Y, Zhang G, Garen A, Song X. Role of human noncoding RNAs in the control of tumorigenesis. Proc Natl Acad Sci.U.S.A. 2009; 106:12956-12961.

42. Tripathi V, Shen Z, Chakraborty A, Giri S, Freier SM, Wu X, Zhang Y, Gorospe M, Prasanth SG, Lal A, Prasanth KV. Long noncoding RNA MALAT1 controls cell cycle progression by regulating the expression of oncogenic transcription factor B-MYB. PLoS Genet. 2013; 9:e1003368-1003385.

43. Lopez-Corral L, Corchete LA, Sarasquete ME, Mateos MV, Garcia-Sanz R, Ferminan E, Lahuerta JJ, Blade J,
Oriol A, Teruel AI, Martino ML, Hernandez J, HernandezRivas JM, et al. Transcriptome analysis reveals molecular profiles associated with evolving steps of monoclonal gammopathies. Haematologica. 2014; 99:1365-1372.

44. Tusher VG, Tibshirani R, Chu G. Significance analysis of microarrays applied to the ionizing radiation response. Proc Natl Acad Sci U.S.A. 2001; 98:5116-5121.

45. Schadt EE, Li C, Ellis B, Wong WH. Feature extraction and normalization algorithms for high-density oligonucleotide gene expression array data. J Cell Biochem Suppl. 2001; Suppl 37:120-125.

46. Chen J, Bardes EE, Aronow BJ, Jegga AG. ToppGene Suite for gene list enrichment analysis and candidate gene prioritization. Nucleic Acids Res. 2009; 37:W305-W311.

47. Subramanian A, Tamayo P, Mootha VK, Mukherjee S, Ebert BL, Gillette MA, Paulovich A, Pomeroy SL, Golub TR, Lander ES, Mesirov JP. Gene set enrichment analysis: a knowledge-based approach for interpreting genomewide expression profiles. Proc Natl Acad Sci U.S.A. 2005; 102:15545-15550.

48. Calura E, Bisognin A, Manzoni M, Todoerti K, Taiana E, Sales G, Morgan GJ, Tonon G, Amodio N, Tassone P, Neri A, Agnelli L, Romualdi C et al. Disentangling the microRNA regulatory milieu in multiple myeloma: integrative genomics analysis outlines mixed miRNA-TF circuits and pathway-derived networks modulated in $\mathrm{t}(4 ; 14)$ patients. Oncotarget 2016; 7:2367-2378. doi: 10.18632/ oncotarget.6151. 